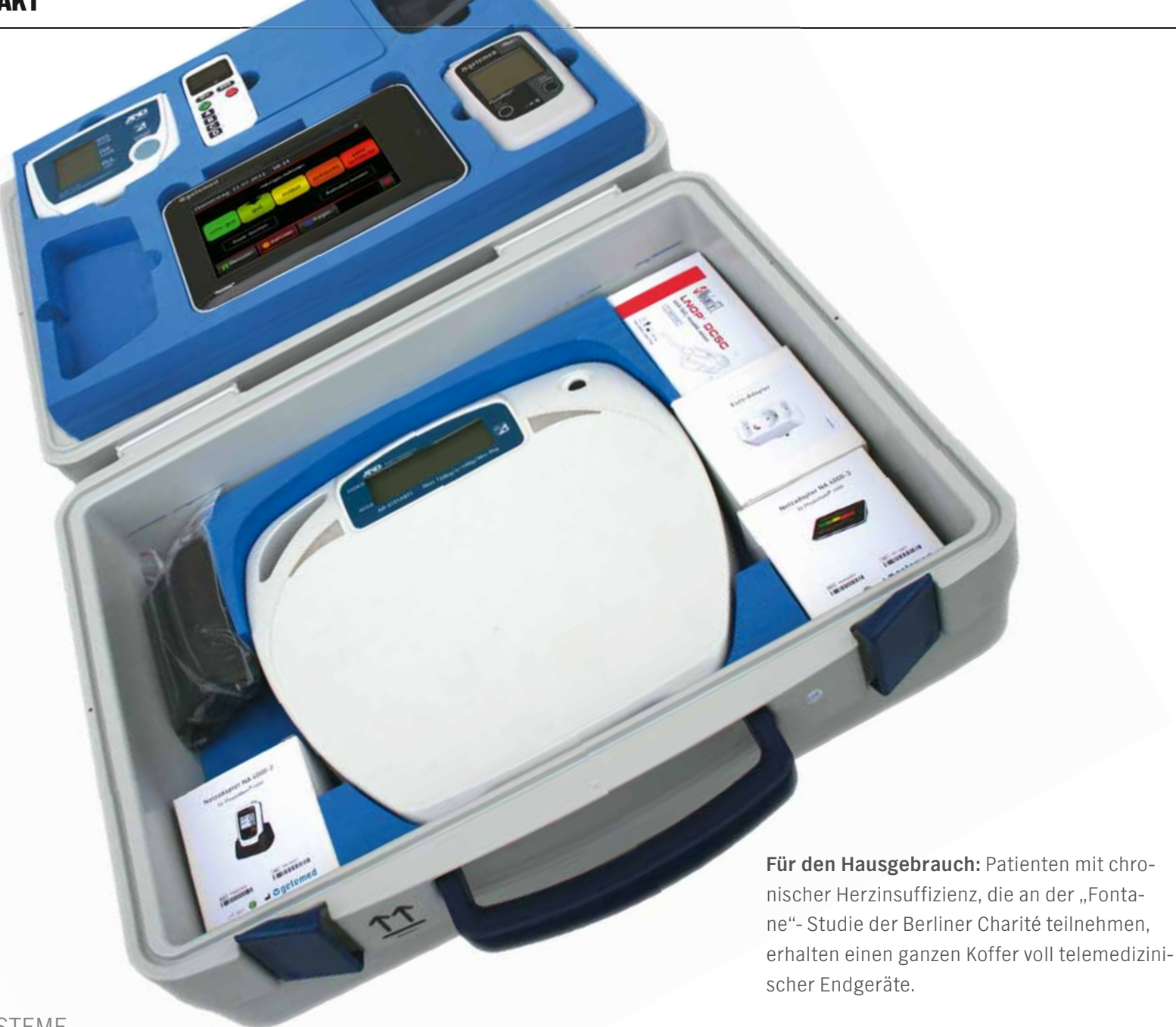

\title{
Doktor mit Flatrate
}

Inzwischen liefert die Industrie ein breit gefächertes Angebot an Telemedizinsystemen. Sie ermöglichen es Ärzten unterschiedlichster Fachdisziplinen, ihre Patienten via Monitor zu behandeln. Selbst der traditionelle Arztbesuch kann durch eine Ferndiagnose ersetzt werden.

D ie ersten Ansätze zur Telemedizin gab es bereits vor zwei Jahrhunderten: Zu dieser Zeit versuchte man, die diagnostischen Maßnahmen über die damals vorhandenen Telefonleitungen einzuleiten. So kam es nach und nach zur Vernetzung der voneinander entfernten medizinischen Standorte. Ein Kanadier Namens Jutras schaffte es bereits im Jahre 1959, zwei Krankenhäuser mit Hilfe eines Teleradiologiesystems zu verbinden. Inzwischen liefert die Industrie ein breites Angebot an Telemedizinsystemen, die für die unterschiedlichsten Anwendungen geeignet sind.
Da es der Technik im Prinzip egal ist, welche medizinische Fachrichtung und welches Krankheitsbild diagnostiziert oder therapiert wird, sind die einzelnen Systeme vielseitig einsetzbar. So sind die verschiedenen mobilen telemedizinischen Arbeitsplätze, die die Hersteller anbieten, für die telemedizinische Unterstützung von Konzilen als auch für die Fernuntersuchung von Patienten in Arztpraxen, Pflegeheimen oder Krankenhäusern geeignet.

\section{Mobile Arbeitsplätze}

Diese Systeme bestehen aus einem fahrbaren Gestell, auf dem neben einem Computer auch ein Monitor mit Tastatur angebracht ist. Durch das Mikrofon und eine hochauflösende Kamera, die sich ebenfalls auf dem mobilen Arbeitsplatz befindet und mit der sich selbst die Diagnose von Pupillen durchführen lässt, ist der Telemediziner in der Lage, in Echtzeit etwa an Tumorkonferenzen oder Fernuntersuchungen teilzunehmen. Für die Übertragung der Daten und Bilder des Patienten an den Telemediziner dient eine verschlüsselte Netzwerkverbindung, die lediglich den Zugriff auf bestimmte Daten des untersuchten Patienten zulässt. Um die 
permanente Netzwerkverbindung zu gewährleisten, können einige Geräte auch per WLAN Daten übertragen. Sie lassen sich entweder direkt oder per Smartcard-Lesegerät an die verschiedenen Klinikinformationssysteme anschließen.

Das Gegenstück dazu ist der sogenannte Befundungsarbeitsplatz, an den der mobile Arbeitsplatz dem Facharzt des Telemedizinzentrums die Patientendaten und das Videobild sendet. Meist ist das der normale Computerarbeitsplatz eines Facharztes, auf dem sich ebenfalls eine Webcam befindet. Einige Hersteller bieten aber auch schon komplett eingerichtete Telearbeitsplätze an, die den Ärzten die telemedizinische Arbeit ergonomisch erleichtern.

\section{Schluckkontrolle aus der Ferne}

Außerdem bietet die Industrie auf spezielle Untersuchung ausgerichtete $\mathrm{Zu}$ satzgeräte an, die sich an die mobilen Arbeitsplätze anschließen lassen. Für neurologische Untersuchungen etwa gibt es ein Diagnosegerät, mit dem sich die Funktion des Schluckmechanismus nach einem Schlaganfall untersuchen lässt. Es besteht aus einer Endoskop-Einheit und einer Speicherund Anzeigeeinheit. Die EndoskopEinheit besteht aus dem Handgriff mit Bedien-Elementen und der Sonde mit Kamera und Beleuchtungssystem und ermöglicht so die Fernuntersuchung des Patienten.

\section{Telemedizin für Zuhause}

Auch für die medizinische Überwachung von chronisch kranken $\mathrm{Pa}$ tienten, die ansonsten für jede Untersuchung zum Hausarzt oder in ein Krankenhaus müssen, bietet die Industrie mittlerweile eine breite $\mathrm{Pa}$ lette telemedizinischer Alternativen an. Solche Patienten erhalten zum

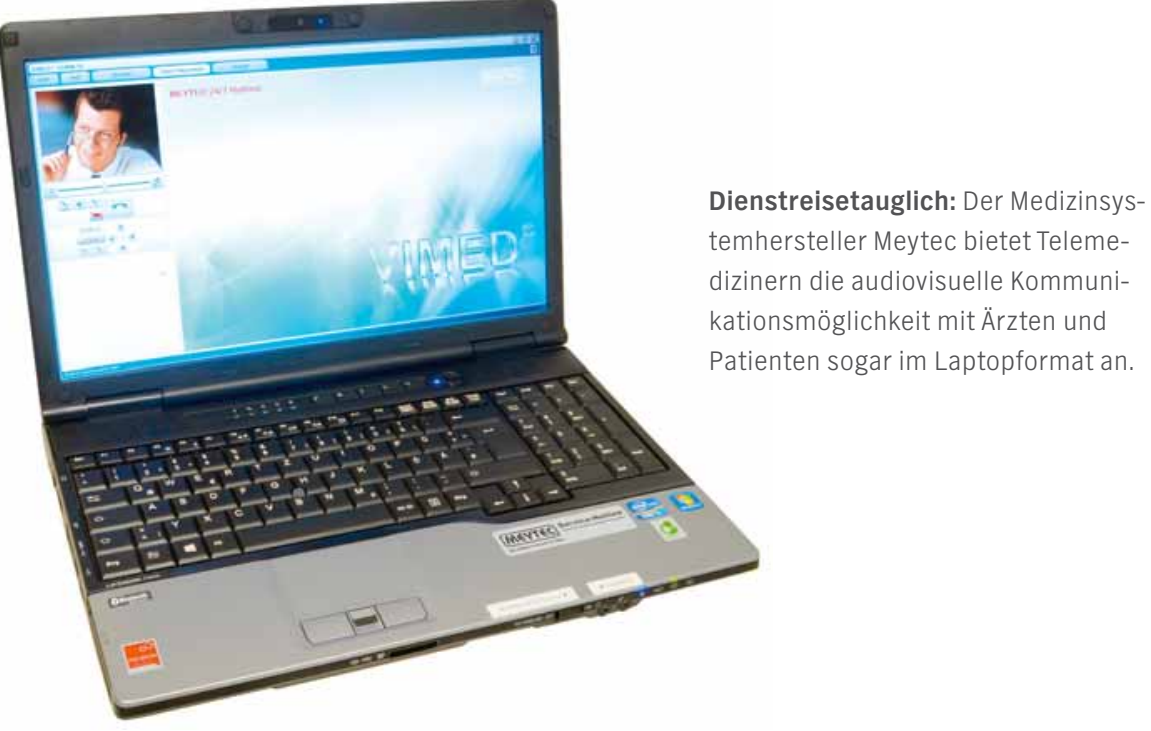

im Zuge des Projekts „Fontane“, das zu den größten Telemedizinstudien zur chronischen Herzinsuffizienz weltweit gehört, einen ganzen Koffer voll sol- 㜽 cher Endgeräte. Sie messen Vitaldaten wie EKG, Sauerstoffsättigung und Blutdruck sowie Angaben etwa zum Gewicht, den aktuellen Befunden oder รั der genauen Medikamenteneinnahme der Patienten. Die technischen Möglichkeiten sind da, man muss sie nur anwenden.

Martin Kucera
So bietet das Zentrum für kardiovaskuläre Telemedizin der Charité Berlin

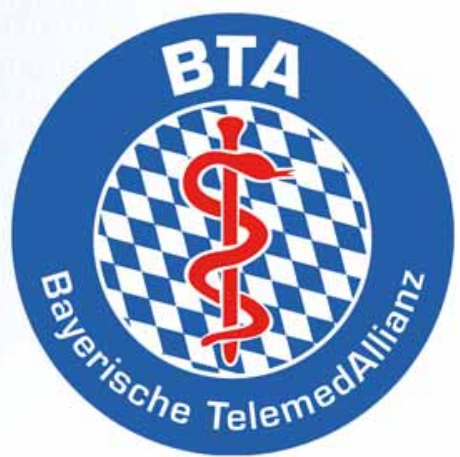

Telemedizin DER Schrittmacher im Gesundheitswesen! 